\title{
جودة التقارير المالية في ضوء ممارسات التحفظ المحاسبي مع دراسة تطبيقية
}

الاكتورة

حسناء عطية

مدرس المحاسبة
أستاذ المحاسبة المساعد

عصام عبد المنعم أحمد

حامد

أستاذ المحاسبة المساعد

كلية التجارة ـ جامعة المنصورة كلية التجارة ـ جامعة المنصورة

$$
\text { الباحث }
$$

علي خيرالله شنين

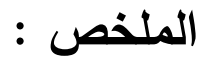

تعد ممارسـات التحفظ المحاسبي مـن الممارسـات المتأصـلة في الممارســة

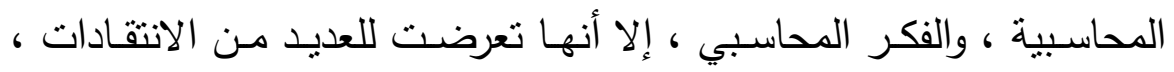

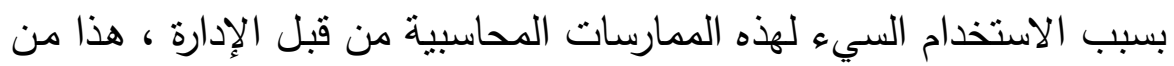

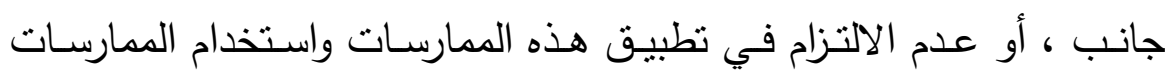

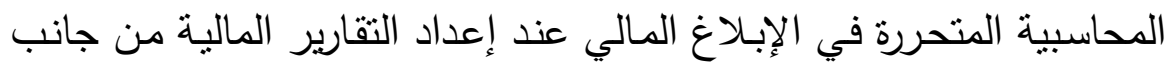

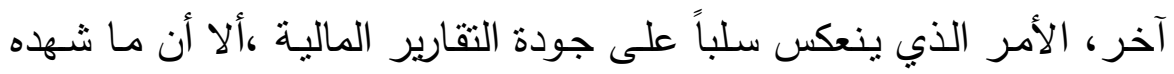

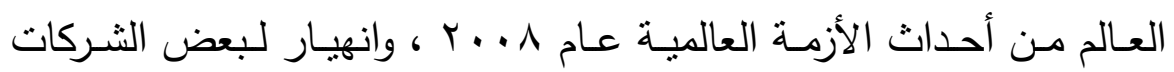

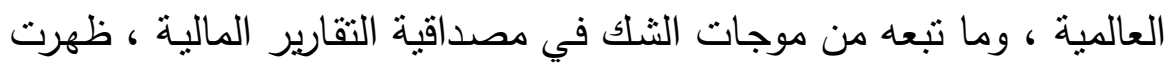

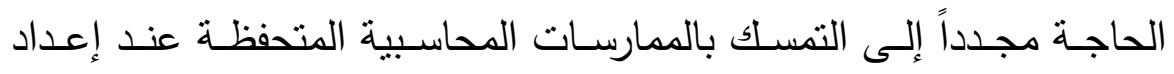

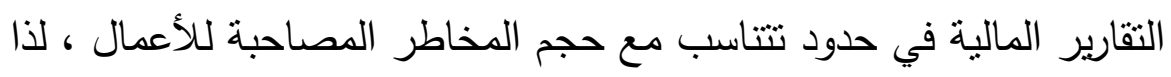


اسـتهدفت هـذه الدراسـة بشـكل رئيسـي توضـيح دور الممارسـات المحاسبية المتحفظة في تحقيق جودة التقارير المالية.

وتوصلت الدراسـة إلى عدة نتائج كان من أهمها وجود علاقة معنويـة ذات

دلالة إحصائية بين الممارسات المحاسبية المتحفظة وجودة التقارير المالية.

وقدمت الدراسـة عدة توصيات كان من أهمها ضـرورة التمسك بالممارسـات

المحاسبية المتحفظـة لمقابلـة العديد مـن المشـاكل سواء المتعلقة بعدم تماثل

المعلومـات بين المنشـأة والأطراف الخـارجيين أو حمايـة المحاسبين ومراقبي

الحسابات من مخاطر التبعات القانونية والقضائية التي يمكن ثتتج من عدم

الالتزام بتطبيق الممارسات المحاسبية المتحفظة ،أضافة لذلك دوره كآلية فعالة

لمواجهة إدارة الأرباح والسياسات الهجومية للإدارة في الإبلاغ عن المعلومات

المحاسبية خصوصـاً المعلومات المتعلقة بالأربـاح ، وضرورة ألزام الثـركات

بمسـتوى مناسـب مـن التحفظ المحاسبي وفقاً لمبـرات تنتاسـب مـع مخـاطر

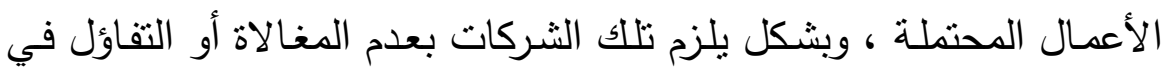

تطبيق ممارسات التحفظ المحاسبي ، لما له من أهمية على دلالة ومصداقية

المعلومـات المحاسبية ، الأمـر الذي يؤدي إلى رفع جودة التقارير الماليـة ،

كما أنه يضمن للشركات المحافظة على مبدأ الثبات والاتساق في السياسات

المحاسبية وعرض المعلومات المحاسبية عند إعداد التقارير المالية.

\section{Abstract:}

accounting conservatism practices is considered as inherent practices in accounting practice and thought the accounting practices. However, it had been to many of the criticisms because of the bad use of these accounting practices by the management, this is a part. On the other part, the noncompliance in the application of these practices and the use of freed accounting practices in financial reporting for the preparation of financial reports which reflect negatively on financial reporting quality. What the world witnessed of the global crisis in 2008 and the collapse of some international companies, and the subsequent doubt of the credibility of financial reports remerged again the need to stick to conservative accounting practices during the preparation of financial reports within the limits commensurate with the risks associated with business. So this study 
was mainly to clarify the role of accounting conservatism practices in achieving financial reporting quality.

The study found several results the significant one is a statistically significant correlation between accounting conservatism practices and financial reporting quality.

The study made several recommendations, most important of which was the need to adhere accounting conservatism practices to pass many of the problems, whether relating to non-uniformity of information between the enterprise and external parties accounting practices, or the protection of accountants and auditors from the risks of legal and judicial consequences that can result from non-compliance by applying accounting conservatism practices.

In addition to its role as an effective mechanism to counter the offensive earnings management and policies of the administration in the reporting of accounting information, especially information relating to profits, And the need to force the companies to be in an appropriate level of accounting conservatism according to the match commensurate with the potential business risks in way that force the companies not exaggerating or optimism in the application of the accounting conservatism practices, because of its importance to the significance and credibility of accounting information which leads to raise financial reporting quality, as it ensures for companies maintain the principle of stability and consistency in the accounting policies and presentation of accounting information during preparation of financial reports.

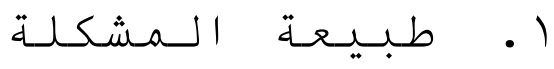

بالنظر إلى الدور الهام الذي تقوم بـه التقارير المالية المنشورة في أسواق رأس المال في تـوفير المعلومـات المحاسـبية التـي ينبغـي أن تعكس حقيقـة الأوضـاع الماليـة للشركات ،أضافة إلى أن حجم التداول للأوراق المالية الخاصة بالثركات ينوقف على لـ له جودة التقارير المالية وقدرتها على جذب الاستثمارات ، الا أن مفهوم جودة التقارير المالية بستتد على مجموعة من المفاهيم والخصـائص المرتبطـة بحمايـة المستثمر ، وإثباع حاجـات مستخدمي معلومات التقارير المالية ، والتي تشكل بمجملها مفهوم الجودة في التقارير الماليـة ، الا أنـه في السنوات الأخيرة خاصـة في ظل الأزمـات المالية ، وظهور العديد من الفضـائح الماليـة في بيئة الأعمـال على مستوى العـالم والتي كـان مـن شـأنها التأثير على مصـداقية معلومـات التقاربر الماليـة ، وبالتـالي 
تقويض تقـة مستخدمي معلومـات التقارير الماليـة ، والتي كان مـن أسبابها سياسـة

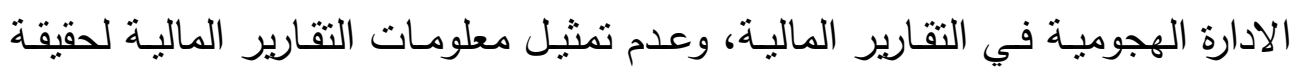
الوضع المالي للشركات ، والنقص في الممارسات المحاسبية المتحفظة التي من شأنها المحافظة على أصول الثركات، ومن هنا يمكن صياغة مشكلة الدراسـة من خلال المال

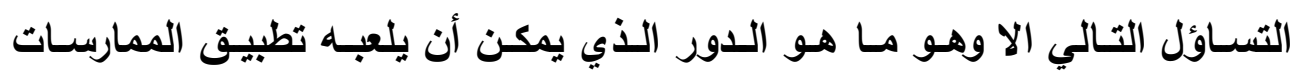

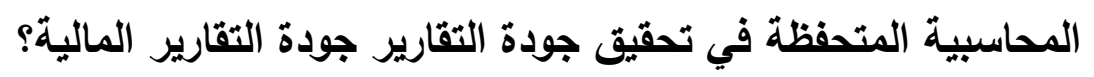

$$
\begin{aligned}
& \text { r- هدف الدراسة : } \\
& \text { تسعى هذه الاراسة إلى تحقيق الأهداف التالية: } \\
& \text { 1- دراسة مفهوم ومكونات ومقاييس التحفظ الدحاسبي. } \\
& \text { r - دراسة مفهوم ومقاييس جودة التقارير المالية. }
\end{aligned}
$$

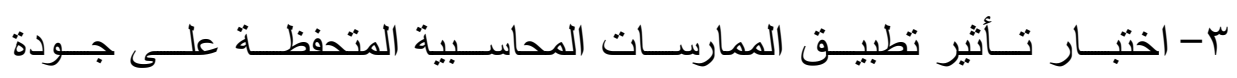

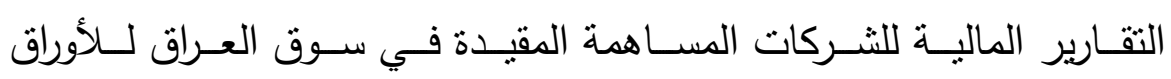
المالية.

\section{فروض الدراسة:}

تحقيقاً لأهداف الدراسة فإنها تقوم على فرضية رئيسية وهي أن التقارير المالية

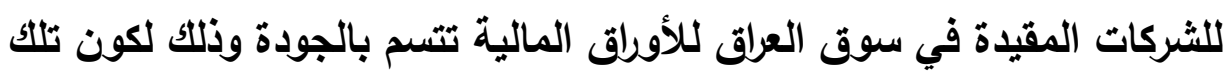

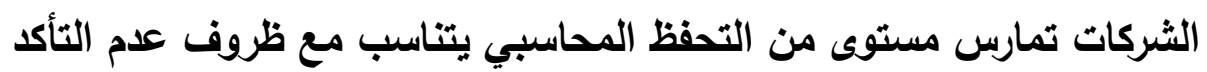
ويمكن صياغة الفرضيات النالية :

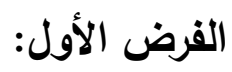
H: لا يوجد ثفاوت في مستوى تطبيق الممارسات المحاسبية المتحفظة في القوائم المالية للثركات المساهمة عينة الدراسة. 
H المالية للشركات المساهمة عينة الدراسة.

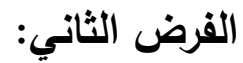

ل ال يوجد تفاوت في مستوى جودة التقارير المالية للثركات المساهمة المقيدة في سوق العراق للأوراق المالية عينة الدراسة. : يوجد تفاوت في مستوى جودة التقارير المالية للشركات المساهمة المقيدة في سوق العراق للأوراق المالية عينة الدراسة.

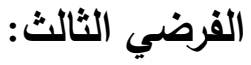

H وتطبيق الممارسات المحاسبية المتحفظة للشركات المساهمة المقبدة في سوق العراق للأوراق المالية عينة الدراسة. H1 توجـد علاقـة معنوبـة ذات دلالــة إحصــائية بـين جـودة التقـارير الماليـة وتطبيـق الممارسـات المحاسـبية المتحفظـــة للثــركات المســاهمة المقيـدة في سوق العراق للأوراق المالية عينة الدراسة.

\section{منهج الدراسة : 2 - من}

أعتمـد الباحـث على المنهجين الاسـتقرائي والتطبيقي مـن خـله الدراسـة النظريـة والتطبيقية فقد تم استقراء وتحليل الأبحاث والدراسات ذات الصلة التي أهتمت بدراسـة موضوع التحفظ المحاسبي وموضوع جودة التقارير ، إما المنهج التطبيقي فتمثل في تجميع البيانات المطلوبة واختبارها في عدد من الشركات المساهمة المدرجة في سوق العراق للأوراق المالية. 


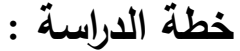

لتحقيق هدف الدراسة يمكن للباحث تقسيم خطة الدراسة على النحو الثالي : 1- ماهية الممارسات المحاسبية المتحفظة.

r- ماهية جودة التقارير المالية.

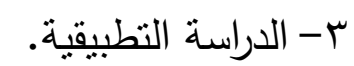

ع - النتائج والتوصيات.

أولاً: ماهية الممارسات المحاسبية المتحفظة:

تعتبر المحاسبة المالية نظام له تأثثر متبادل مع البيئة المحيطة بهه وكما هو معروف

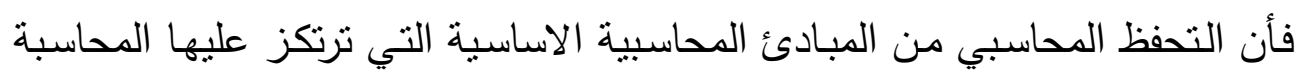
المالية وأقدمها فأن التحفظ المحاسبي ينأثر بمجموعة من العوامل المحيطة به، فضانلاً عن تأثيره الكبير على دلالة ومصداقية القوائم المالية سواء كأن ذلك سلباً أو ايجاباً

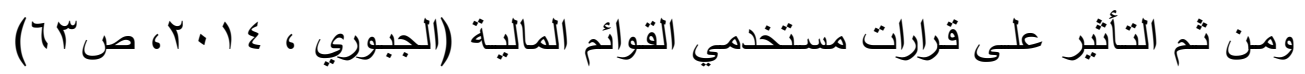

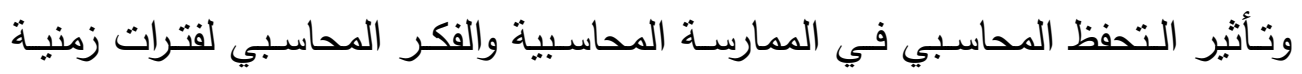

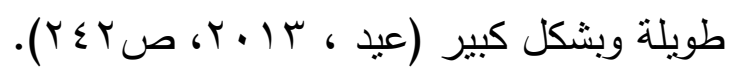

Watts ,2003a,p.2 ; Ball et ( بعد التحفظ المحاسبي مبدأ اساسي في المحاسبة ) ويمثل أقدم المبادئ المحاسبية ، اذ أعتاد الرواد الأوائل في مي المحاسبة على استخدام مصطلح الحيطة والحذر الذي يشير إلى التحفظ الدحاسبي ، والمقصود به تسجيل الخسائر الاقتصادية قبل وقوعها والاعتراف بها في القوائم المالية

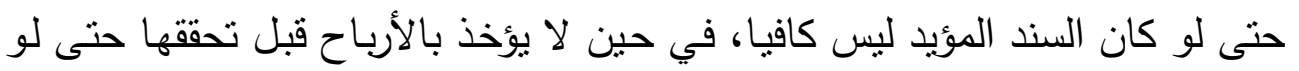

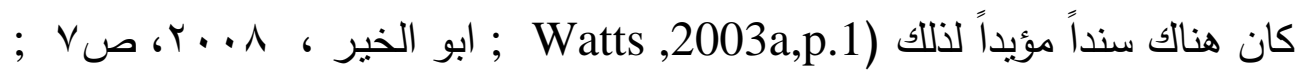

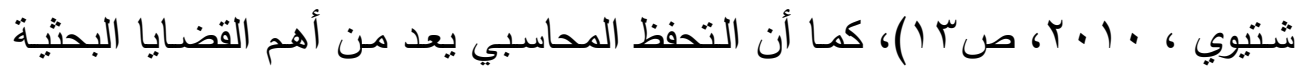

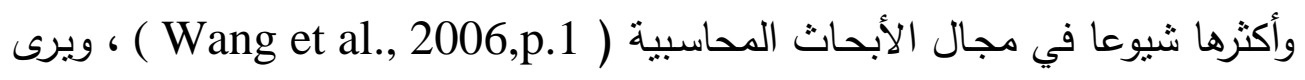

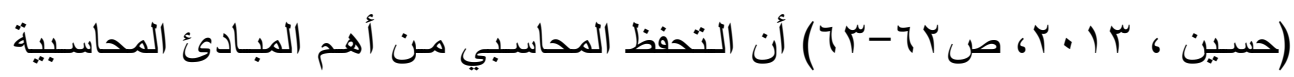


التي لها تأثير على ملائمسة وصدق المعلومات المحاسبية الواردة في التقارير المالية

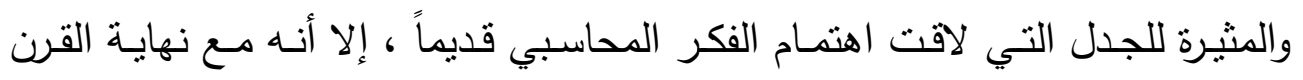

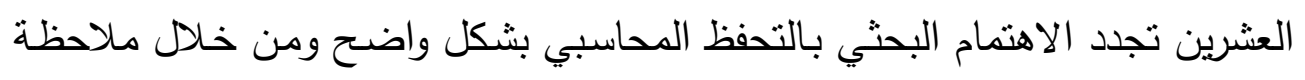
العديد من الدراسات النظرية والتطبيقية الحديثة المتعلقة بالتحفظ المحاسبي ( الرشيدي النئي

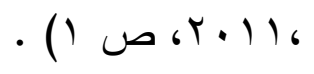

ويمثل التحفظ المحاسبي بشكل أكثر دقة ميل المحاسبين عندما يواجهون حالة

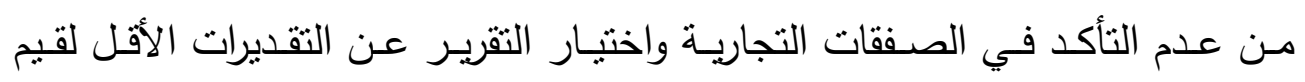

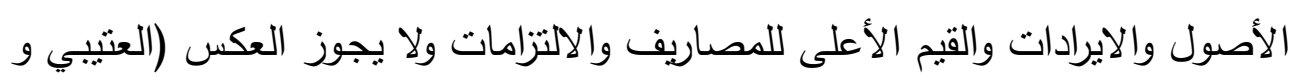

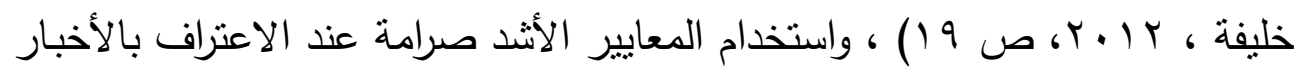

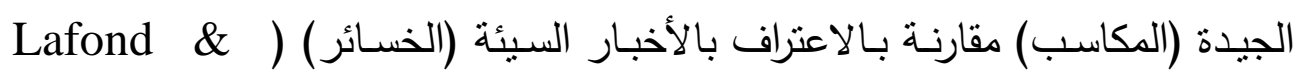

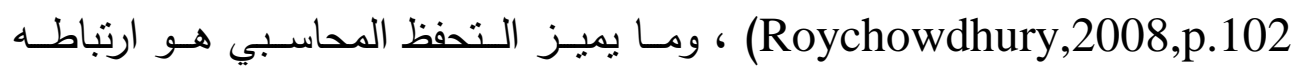

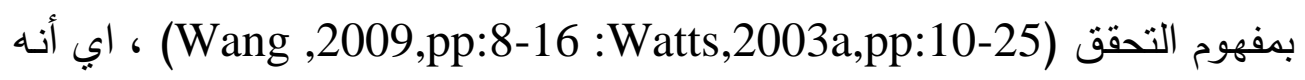

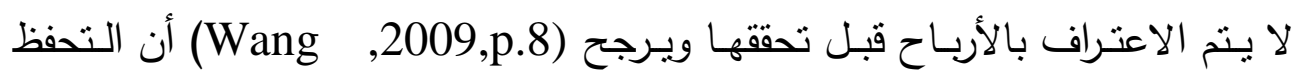
المحاسبي أحد أساسيات مبدأ التحقق.

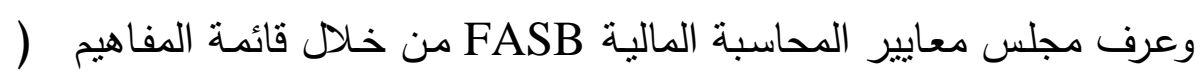
(Concepts2

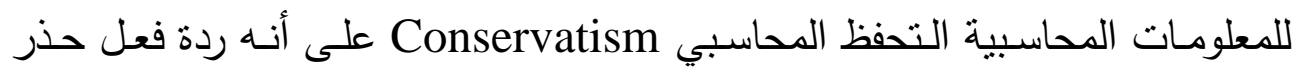

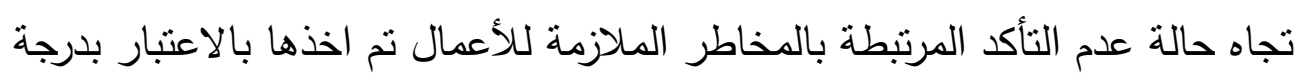
كافية.

ويمثل تعريف (1924, 1924) اقدم التعريفات الخاصة بالتحفظ المحاسبي بالقول

المأثور عدم توقع أية أرباح ولكن نتوقع كل الخسائر ، (Watts,2003a,p.1) . أما في المنظور المعاصر فقد عرف (Basu, 1997, p.4 ) التحفظ المحاسبي على أنه ميل المحاسبين للحصول على درجة عالية من التحقق للاعتراف بالأخبار الجيدة

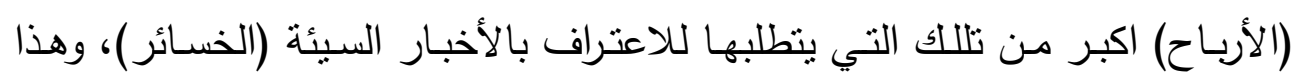


التحقق غير المتماثل يؤدي إلى تخفيض مستمر لقيمة صافي الأصول وتوليد أرباح ويعكس الأخبار السيئة بطريقة أكثر توقيتاً من الأخبار الجيدة.

أما (Givoly \& Hayn, 2000, pp.291-292) فقد عرفا التحفظ المحاسبي على أنه معيار للاختيار بين المبادئ والسياسات المحاسبية التي تقود إلى تخفيض الأرباح

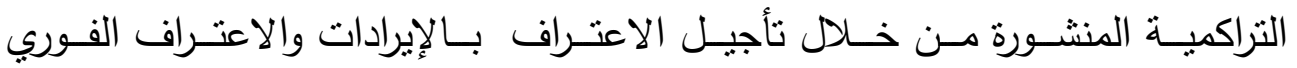

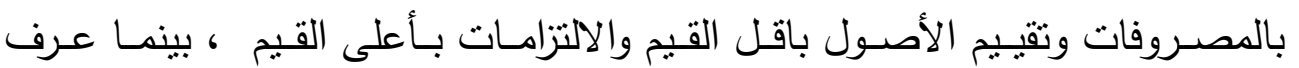
2002,p.239)(Penman \&Zhang ,

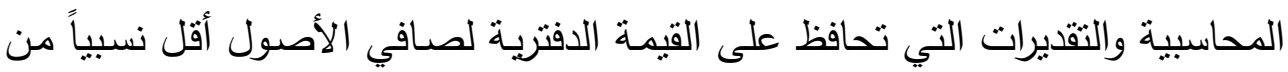
قيمتها السوقية ، فعلى سبيل المثال طريقة (LIFO ) للمحاسبة عن المخزون تكون متحفظة بالنسبة لطريقة ( FIFO) اذا كانت تكاليف المخزون في ارتفاع ، بينما

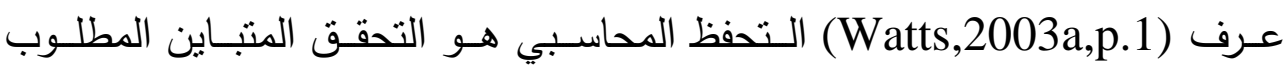

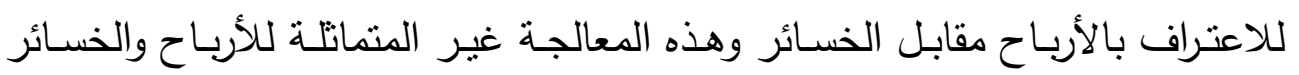

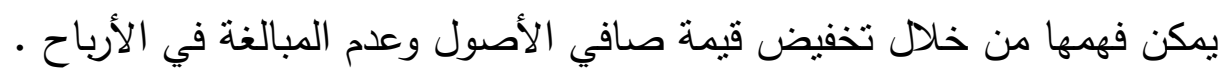

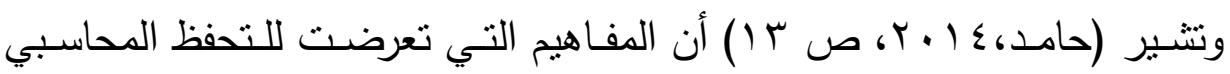

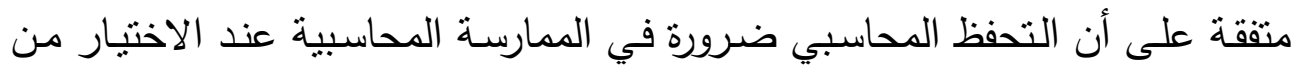

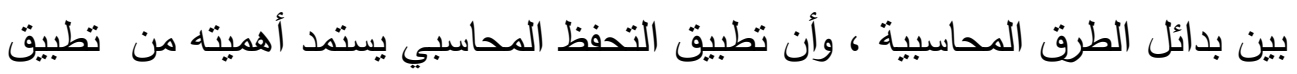

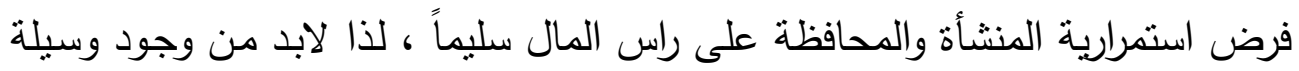
للتصدي للتغير المستمر في دوافع معدي القوائم المالية في اختيار بديل دون الآخر

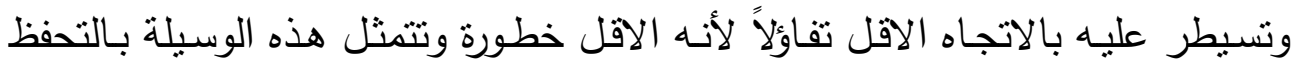

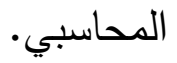
ويرى الباحث من خلال استعراض تعريفات التحفظ أنه لا يوجد تعريف محدد متفق

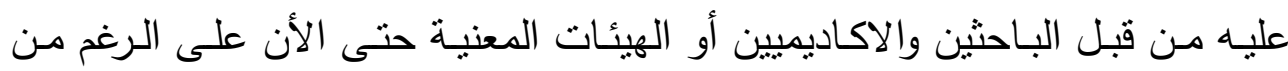

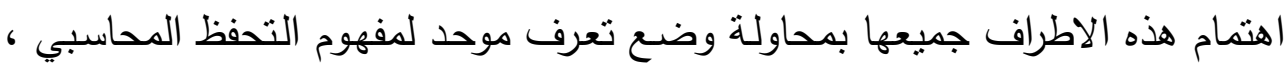
كما أن التحفظ المحاسبي هو مبدأ يضم مجموعة من الممارسـات المحاسبية التي وني 
تستخدم في توقيتات ، وحالات مختلفة ، ومحددة ، والهدف منها الحفاظ أو زيادة قيمة الثركة ، وحماية حقوق جميع الأطراف الداخلية والخارجية في ظل حالة عدم التأكد التي تسبب مخاطر تلازم الاعمال التجارية. ثانياً: ماهية جودة التقارير المالية:

أصسبحت جـودة التقارير الماليـة ومحساولات التطـوير الخاصـة بهـا لكي ترتقـي بالحاجـات المتوخـاة منها مـن قبـل مسـتخدمي الثقارير الماليـة بشـكل سـليم وفعـال

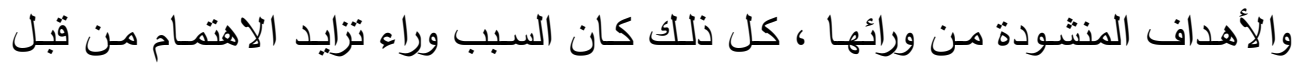
العديد من الباحثين بشأن مفهوم الجودة في التقارير المالية. فانهيار الثقة بالتقارير المالية التي تعد من أهم مخرجات النظام ومصدر رئيسي

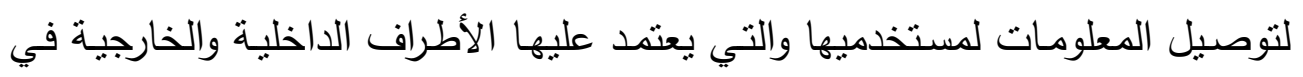
اتخاذ القرارات الاقتصادية ، لذا كان من الضروري محاولة إعادة نقة المستخدمين

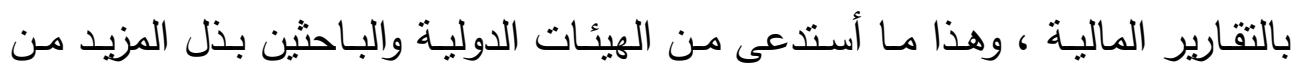
الجهود التي تصب في حماية حقوق ومصالح جميع الاطراف . وتعد النقارير المالية المنتج النهائي للعمل المحاسبي وهي وسيلة الإفصـاح عن

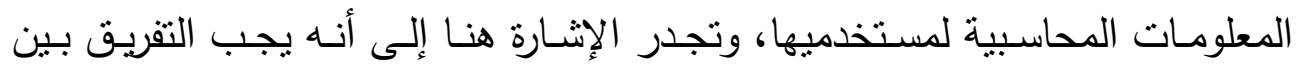

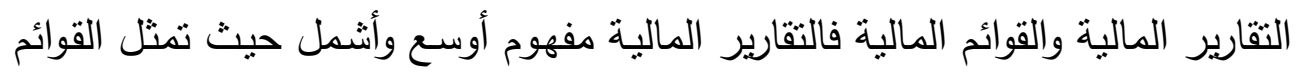

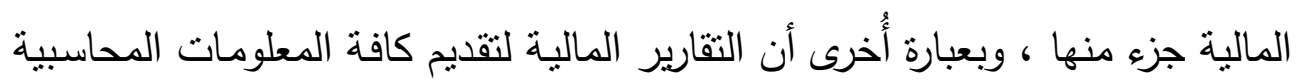

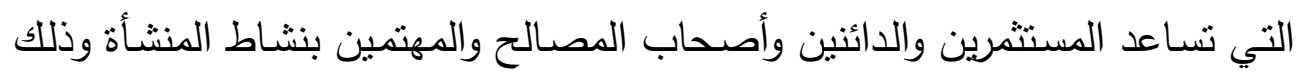

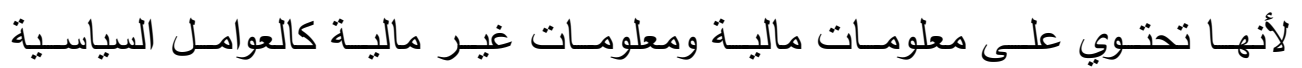

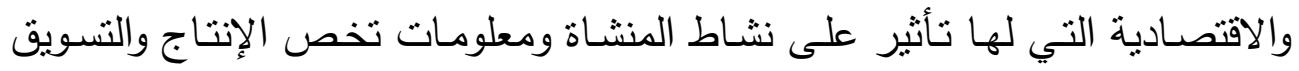
وغير ذللك ، أما القوائم المالية فهي جزء حئ حيوي من التقارير المالية حيث أنها تمثل

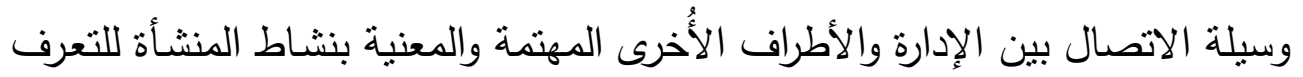

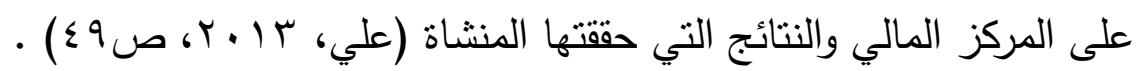


تعرف الجودة على أنها مدى ملائمـة المنتج أو الخدمـة لإثـباع رغبـات المستخدم

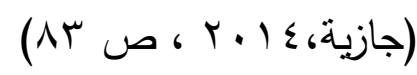

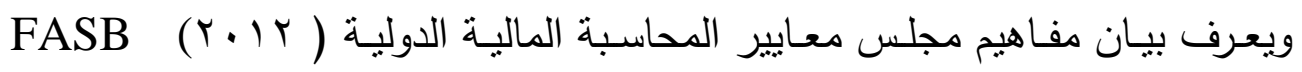
القفرة r concept statement (2) جودة الثقارير المالية بأنها الخصائص النوعية للمعلومات المحاسبية مبوبة بشكل تتظيم هرمي تتكون من خاصيتين رئيسيتين وهما

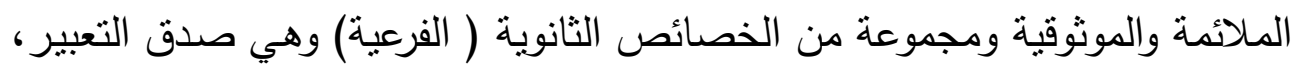

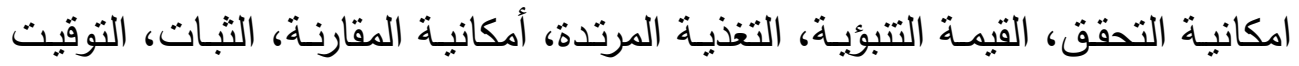

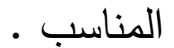

كما عرفها اتحاد المحللين الماليين بأنها مدى تفصيل ووضوح المعلومات الدحاسبية وتقديمها في الوقت المناسب ، كما عرفها المعهد الامريكي للمحاسبين القانونيين ALCPA

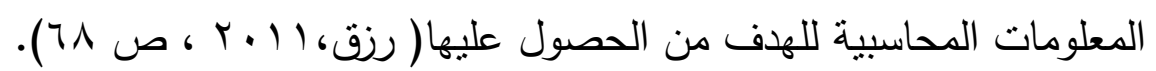
أيضا عرف (Jonas \& Blanchet, 2000,pp:355-356) جودة التقارير المالية على أنها مدى توافر الخصائص الأساسية للمعلومات المحاسبية التي تضمن تحقيق الأهداف الأساسية للتقارير المالية التي تشبع حاجة مستخدمي التقارير المالية وتضمن لتصنيه حماية المستثمرين.

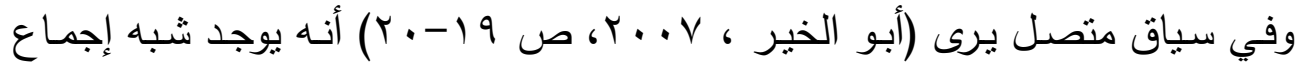

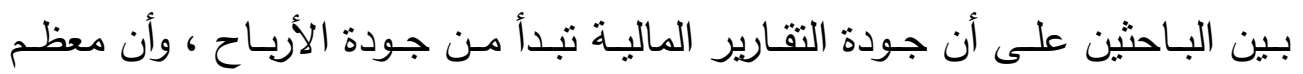
الباحثين استخدما مقاييس مرتبطة بجودة الأرباح المحاسبية في قياس جودة التقارير

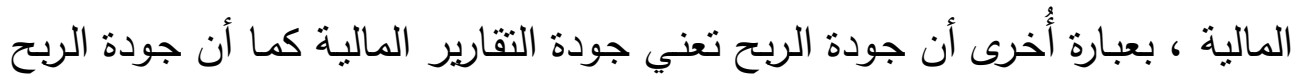

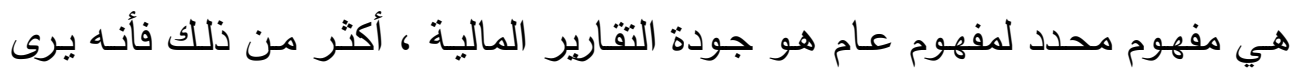
البعض أن جودة الأرباح المحاسبية من المرجح أن نتوب عن جود هودة التقارير المالية

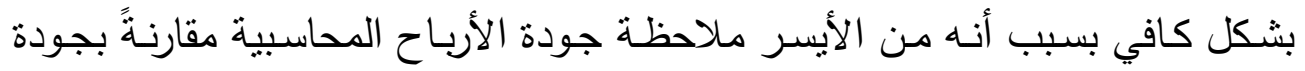


التقارير المالية من الناحية الاحصائية ، وحتى أن لم تكن تكفي فأنها تعتبر من أجود المؤشرات واكثرها أهمية لنتائج عملية إعداد التقارير المالية.

ويرى الباحث أنه إذا ما أتسمت الأرباح بالقدرة على التتبؤ وأمانة العرض والقدرة على تحقيق سمة الاستمرارية فأنها تعد من أبرز المؤشرات لتحقيق جودة التقارير المالية .

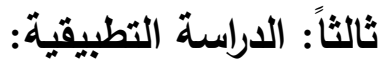

\section{مجتمع وعينة الاراسة}

يتكون مجتمع الدراسة من جميع الثركات المساهمة الدرجة في هيئة سوق العراق

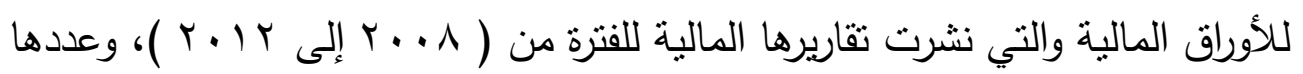

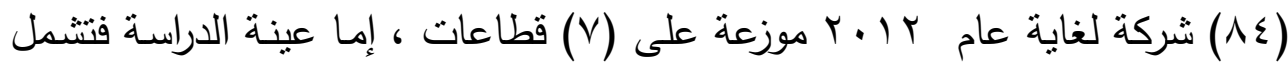

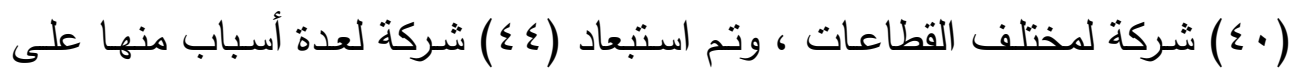

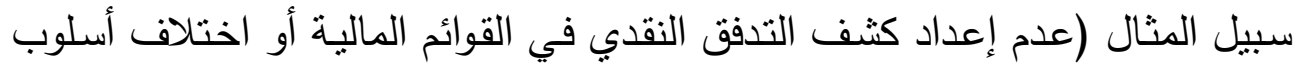
عرض البيانات المالية أو عدم توفر البيانات المالية لبعض السنوات لثركات لثرات معينة)

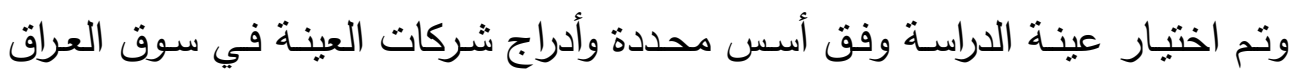

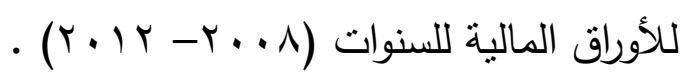
لغرض لقياس متغيرات الدراسة المتمثلة بممارسات ممارسات التحفظ المحاسبي وجودة

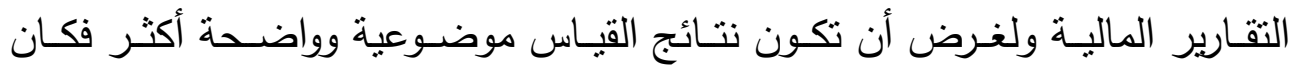

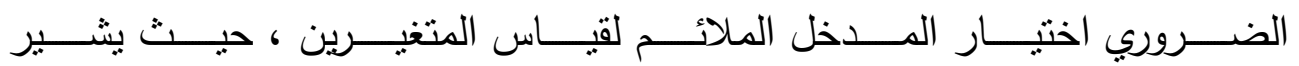
(watts,2003a:Ball\&shivakumar,2005) تتعكس في جودة التقارير المالية وهو يعتبر من المؤشرات الهامة في جودة التقارير

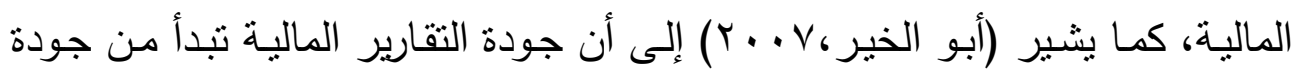

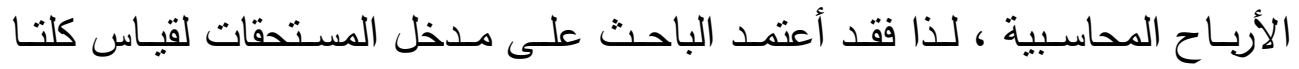
المتغيرين وهذا مـا يميز هذه الدراسـة عن الدراسـات السـابقة وقد تم قياس ممارسات

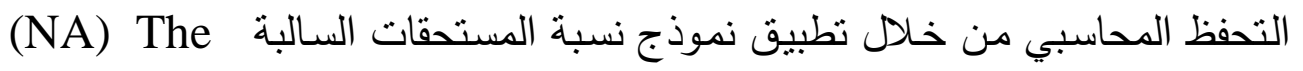


(Givoly\&Hayn,2000) الذي قدمته دراسة) Negative Accruals Measure

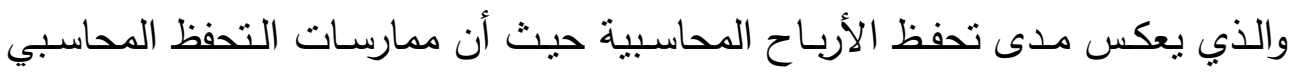
تعمل على تأجيل الاعتراف بالأرباح وتعجيل الاعتراف بالخسائر ومن خلال تكرار

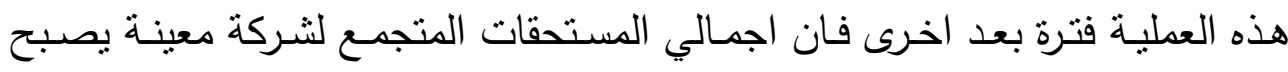

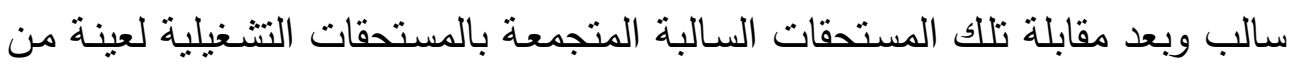
الثركات كانت النتيجة الختامية سالبة معتبرين نلك النتيجة مؤشرا على زيادة ممارسة

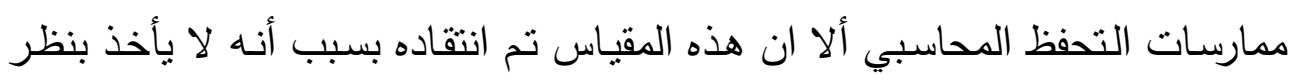

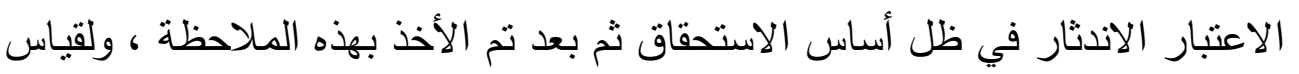

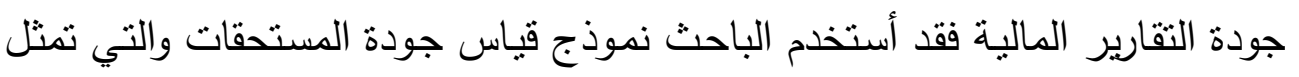

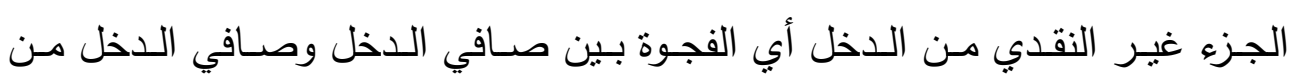

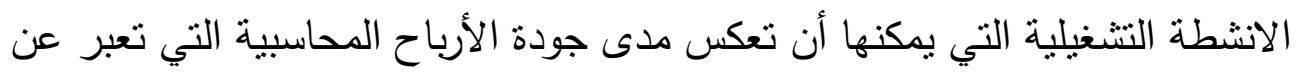
جودة التقارير المالية وقد أستخدم الباحث نموذج (Jones,1991) المعدل من قبل فيل فئل (Dechow,1995) لقياس جودة الثقارير المالية من خلال جودة المستحقات.

• قياس ممارسات التحفظ المحاسبي وفقاً لنموذج نسبة المستحقات السالبة :(NA)

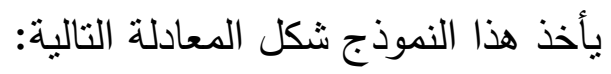
$\mathbf{N A}_{\text {it }}=\mathbf{T A C C}_{\text {it }} / \mathbf{N}_{\text {it }}$

حيث أن: t. تمثل ممارسات التحفظ المحاسبي للثركة i في السنة :NA tt تمنل أجمالي المستحقات الكلية للشركة i في السنة TACC t صافي الدخل زائداً مصروف الاندثار والإطفاء للشركة i في السنة it ويمكن حساب أجمالي المستحقات الكلية بطريقة الميزانية وفقا للمعادلة التالية: 
TACCit $=\Delta \mathrm{CA}-\Delta \mathrm{Cash}-\Delta \mathrm{CL}+\Delta \mathrm{STDEP}-$ Dep

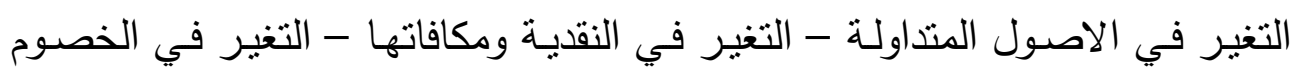

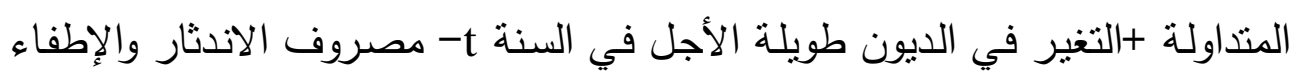

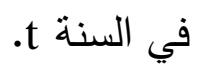

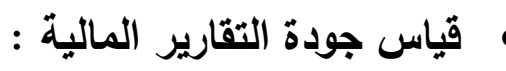

تم قياس جودة التقارير المالية باستخدام نموذج (Jones,1991) المعدل من قبل (Dechow,1995)

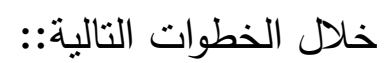

1- احتساب المستحقات الاجمالية من الانشطة التشغيلية.

ץ- الحصول على الثوابت من نموذج انحدار نموذج (Jones,1991). r- احتساب المستحقات غير الاختيارية من خلال تعويض المعاملات التي تم الحصول عليها في فقرة (Y) في مقياس(Jones,1991) المعدل.

ع - ينتم احتسـاب المستحقات الاختياريـة مـن خـلال طـرح المستحقات غير الاختيارية من أجمالي المستحقات.

1- TACCit $=\Delta \mathrm{CA}-\Delta \mathrm{Cash}-\Delta \mathrm{CL}+\Delta \mathrm{STDEP}-$ Dep

2- $\frac{\text { TACCit }}{\text { Ait-1 }}=\beta 1\left(\frac{1}{\text { TAit-1 }}\right)+\beta 2\left(\frac{\Delta \text { REV it }-\triangle \text { RECit }}{\text { TAit-1 }}\right)+\beta 3\left(\frac{\text { PPEit }}{\text { TAit-1 }}\right)+$ Eit

3- NDACCit $=\beta 1\left(\frac{1}{\text { TAit-1 }}\right)+\beta 2\left(\frac{\triangle \text { REVit }-\triangle \text { RECit }}{\text { TAit-1 }}\right)+\beta 3\left(\frac{\text { PPEit }}{\text { TAit-1 }}\right)+$ Eit

4- DACCit $=$ TACCit - NDACCit

i تمثل أجمالي المستحقات الكلية من الأنشطة التشغيلية للثركة TACCit في السنة t. :تمنل أجمالي الأصول للسنة السابقة (أول المدة). . تمنل المستحقات غير الاختيارية للشركة i في السنةt:NDACC 
ت تمتل المستحقات غير الاختبارية للثركة i في السنةt: DACC

t تمنل التغير في إيرادات (المبيعات) للثركة i في السنة .t تمنل التغير في الذمم المدينة للثركة i في السنة t. تمنل أجمالي الأصول الثابتة للشركة i في السنة: PPE

N : تمثل المستحقات غير الاختيارية. : B $B_{1}, \beta_{2}, \beta_{3}$

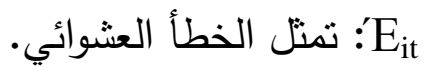

\section{• عرض وتفسير نتائج الاختبارات الاحصائية}

تم استخدام البرنامج الإحصائي (SPSS) في الاختبارات الإحصائية للتعامل

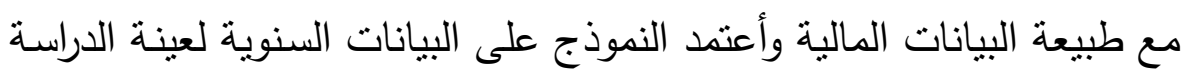

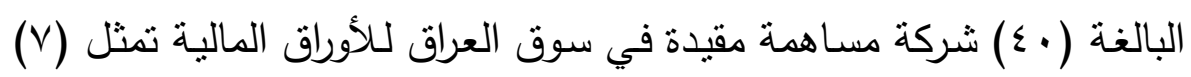

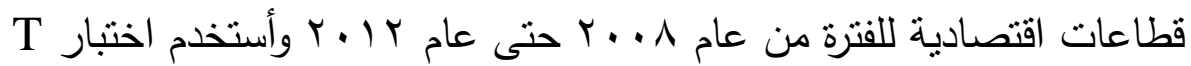
للفرضيتين الأولى والثانية وأستخدم الباحث أسلوب (One sample test) الانحدار الخطي المتعدد لاختبار العلاقة ما بين تطبيق الممارسات المحاسبية ولئية المتحفظة وجودة جودة التقارير المالية (Regression linear stepwise)

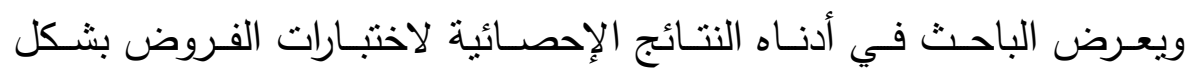
تفصيلي:

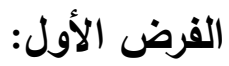

H: لا يوجد تفاوت في مستوى تطبيق الممارسات المحاسبية المتحفظة في القوائم المالية للثركات المساهمة عينة الدراسة.

H1 يوجد تفاوت في مستوى تطبيق الممارسات المحاسبية المتحفظة في القوائم المالية للشركات المساهمة عينة الدراسة. 


\section{جدول رقم (1) اختبار T لعينة واحدة}

\begin{tabular}{|c|c|c|c|c|c|c|}
\hline \multicolumn{7}{|c|}{ One-Sample Test } \\
\hline \multirow{3}{*}{ التحمارسات } & \multicolumn{6}{|c|}{ Test Value $=0$} \\
\hline & $\mathbf{T}$ & DF & $\mathbf{N}$ & $\begin{array}{c}\text { Mean } \\
\text { Difference }\end{array}$ & $\begin{array}{c}\text { Std. } \\
\text { Deviation }\end{array}$ & Sig \\
\hline & $-.571-$ & 39 & 40 & $-1.2253682-$ & 13.5690018 & .571 \\
\hline
\end{tabular}

(One simple test) T بتضـح من خلال الجدول أعلاه أنه تم استخدام اختبار لغرض معرفة ما إذا كان هناك فروق معنوية في مستوى تطبيق الممارسات المحاسبية

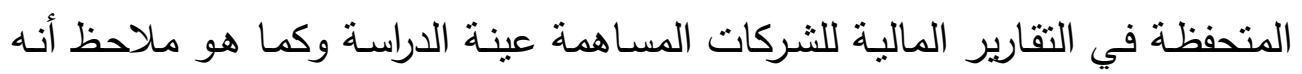

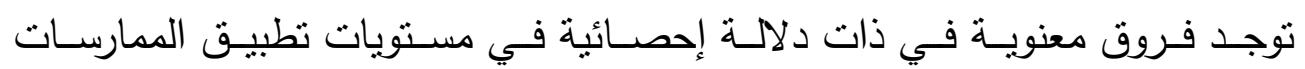

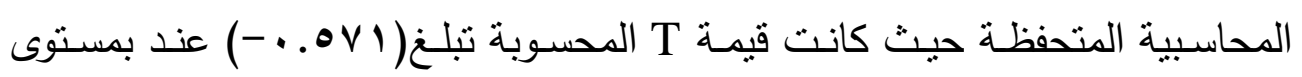

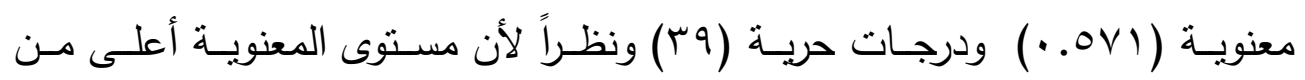

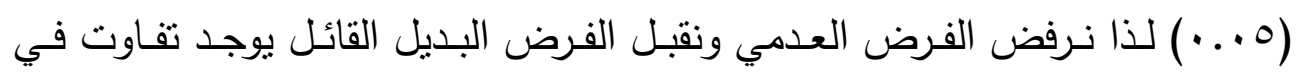

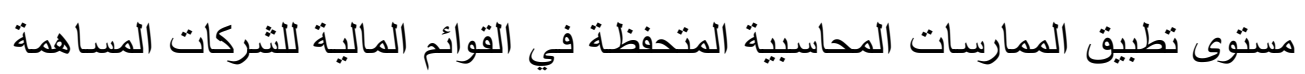
عينة الدراسة.

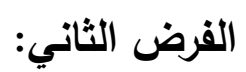
سوا سوق العراق للأوراق المالية عينة الدراسة.

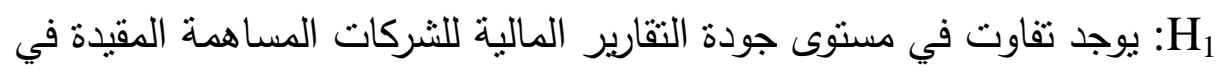
سوق العراق للأوراق المالية عينة الدراسة. جدول رقم (Y) اختبار T لعينة واحدة

\begin{tabular}{|c|c|c|c|c|c|c|}
\hline \multicolumn{7}{|c|}{ One-Sample Test } \\
\hline \multirow{3}{*}{ الاختيارية } & \multicolumn{6}{|c|}{ Test Value $=0$} \\
\hline & $\mathbf{T}$ & DF & $\mathbf{N}$ & $\begin{array}{c}\text { Mean } \\
\text { Difference }\end{array}$ & $\begin{array}{c}\text { Std. } \\
\text { Deviation }\end{array}$ & Sig. \\
\hline & -1.147 & 39 & 40 & $-.0249406-$ & .1375785 & .259 \\
\hline
\end{tabular}


(One simple test) T يتضـح من خـل الجدول أعلاه أنه تم استخدام اختبار لغرض معرفة ما إذا كان هنالك فروق معنوية في مستوى المستحقات الاختيارية في

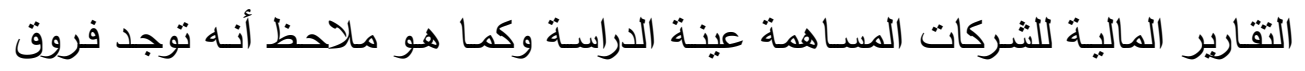

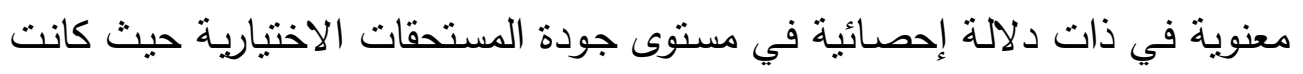

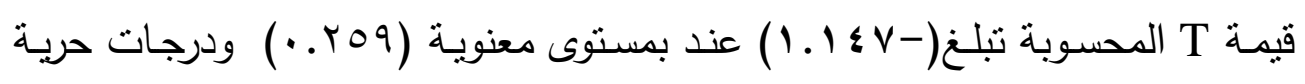

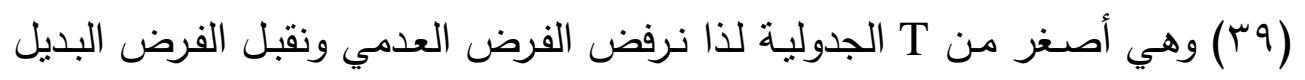
القائل يوجد تفاوت في مستوى تطبيق الممارسـات المحاسبية المتحفظة في القوائم المالية للثركات المساهمة عينة الدراسة.

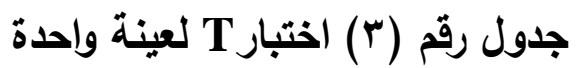

\begin{tabular}{|c|c|c|c|c|c|c|}
\hline \multicolumn{7}{|c|}{ One-Sample Test } \\
\hline \multirow{3}{*}{ الاخستحقات غير } & \multicolumn{6}{|c|}{ Test Value $=0$} \\
\hline & $\mathbf{T}$ & DF & $\mathbf{N}$ & $\begin{array}{c}\text { Mean } \\
\text { Difference }\end{array}$ & $\begin{array}{c}\text { Std. } \\
\text { Deviation }\end{array}$ & Sig. \\
\hline & $-1.170-$ & 39 & 40 & $-.0436167-$ & .2357121 & .249 \\
\hline
\end{tabular}

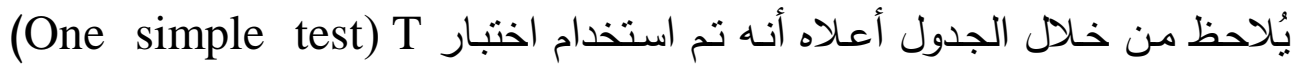
لغرض معرفة ما إذا كان هناك فروق معنوية في مستوى المستحقات غير الاختبارية

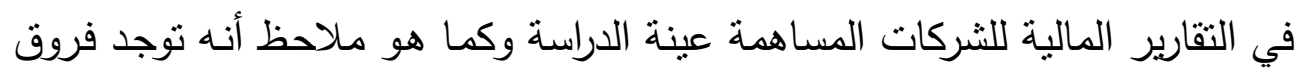

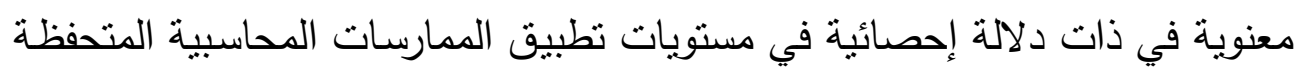

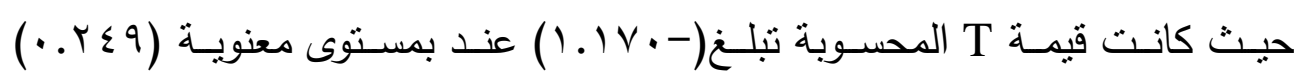

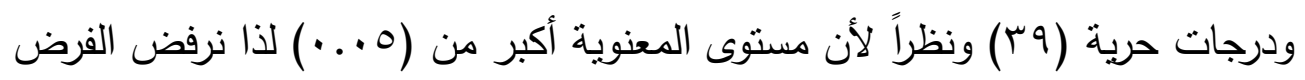

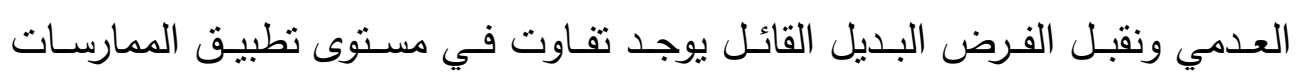
المحاسبية المتحفظة في القوائم المالية للشركات المساهمة المقيدة في سوق العراق للأوراق المالية عينة الدراسة. 


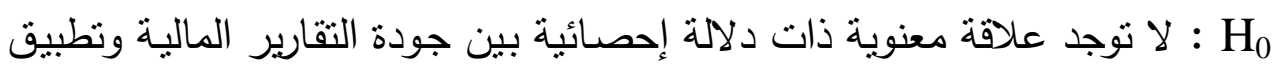
الممارسات المحاسبية المتحفظة للشركات المساهمة المقيدة في سوق العراق للأوراق المالية عينة الدراسة.

: توجد علاقـة معنويـة ذات دلالــة إحصـائية بـين جـودة التقارير الماليـة وتطبيـق الممارسات المحاسبية المتحفظة للشركات المساهمة المقيدة في سوق العراق للأوراق المالية عينة الدراسة.

جدول رقم ( ) مصفوفة ارتباط بيرسون لمتغيرات النموذج

\begin{tabular}{|c|c|c|c|c|}
\hline \multicolumn{5}{|c|}{ Correlations } \\
\hline & & ممارسات التحفظ & المستحقات غير & الاختيارية \\
\hline \multirow{3}{*}{ Pearson Correlation } & ممارسات التحفظ المحاسبي & 1.000 & .957 & .785 \\
\hline & المستحقات غير الاختيارية & .957 & 1.000 & .901 \\
\hline & المستحقات الاختيارية & .785 & .901 & 1.000 \\
\hline \multirow{3}{*}{ Sig. } & ممارسات التحفظ المحاسبي & . & .000 & .000 \\
\hline & المستحقات غير الاختيارية & .000 & . & .000 \\
\hline & المستحقات الاختيارية & .000 & .000 & \\
\hline \multirow{3}{*}{$\mathbf{N}$} & ممارسات التحفظ المحاسبي & 39 & 39 & 39 \\
\hline & المستحقات غير الاختيارية & 39 & 39 & 39 \\
\hline & المستحقات الاختيارية & 39 & 39 & 39 \\
\hline
\end{tabular}

يتضح من خلال الجدول رقم (Y) ( الخاص بمصفوفة الارتباط بين متغيرات جودة التقارير المالية المتمثلة في المستحقات الاختيارية والمستحقات غير الاختيارية مع ممارسات التحفظ المحاسبي أنه كانت العلاقة علاقة ارتباط موجبة بين المتغيرات وكما يُلاحظ أن درجة ارتباط ممارسات التحفظ المحاسبي مع المستحقات غير الاختيارية كانت تمنل (90,v . • ) وبمستوى معنوية ( . . •) ، إلى جانب ذللك فقد كانت درجة ارتباط ممارسات التحفظ المحاسبي مع المستحقات الاختيارية تمنل 


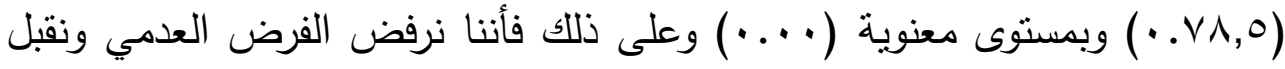
الفرض البديل القائل توجد علاقة معنوية ذات دلالة إحصائية بين جودة التقارير المالية وتطبيق الممارسات المحاسبية المتحفظة للشركات المساهمة عينة الدراسة وتجدر الإشارة الى أن برنامج التحليل قام باستبعاد أحدى الثركات التي كانت قيم بياناتها شاذة وفقاً للحدود المعيارية للمعيار الاحصائي Tolerance الذي يقبل انحراف عن حدود معايرته الاحصائية وكدد أقصى ·(\% وهي كانت تمنل .$(\% \backslash \wedge . \vee)$

جدول رقم (ه) معاملات الانحدار للمتغيرات المستقلة على ممارسات التحفظ المحاسبي

\begin{tabular}{|c|c|c|c|c|c|c|c|c|c|}
\hline \multicolumn{2}{|c|}{ التقليرات } & \multirow{2}{*}{ الاحدارل } & \multirow{2}{*}{ الآحدارج } & \multirow{2}{*}{ Sig. } & \multirow{2}{*}{ DF } & \multirow{2}{*}{$\mathbf{F}$} & \multirow{2}{*}{$\mathbf{R}^{2}$} & \multirow{2}{*}{$\mathbf{R}$} & \multirow{2}{*}{ المتغيرات } \\
\hline Sig. & $\mathbf{T}$ & & & & & & & & \\
\hline I & $\cdot v \leqslant \varphi_{-}$ & $\begin{array}{r}-.94 \\
4\end{array}$ & الثابت & \multirow{3}{*}{$\because \ldots$} & 1 & \multirow{3}{*}{$r . \wedge \wedge 1$} & \multirow{3}{*}{$\because .9 \leqslant v$} & \multirow{3}{*}{$.9 \vee \mathrm{V}$} & \multirow{3}{*}{ التمارفي } \\
\hline . & 10.04 & ro.rA & الاختيريرية & & 4 & & & & \\
\hline$\ldots$ & בה היד. & r. & الاختيتريةتة & & rv & & & & \\
\hline
\end{tabular}

لقد تم استخدام Regression linear stepwise لغرض معرفة تأثنر خصائص جودة التقارير الماليـة على الممارسـات المحاسبية المتحفظـة فقد تم أدخـال المتغيـر التابع والمتمثل في ممارسات التحفظ المحاسبي والمتغيرات المسنقلة المتمثلة في المستحقات الاختياريـة والمستحقات غير الاختياريـة ومسن الجدول اعـلاه بمكنتـا ملاحظـة أن قيمـة

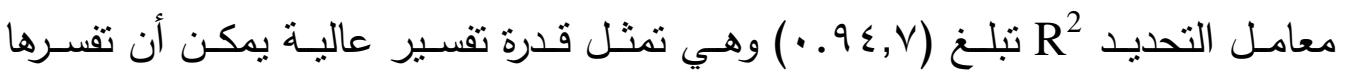
المتغيرات المستقلة (المستحقات غير الاختيارية والمستحقات الاختيارية) للتغيرات التي من الممكن أن تحدث للمتغير التابع (ممارسـات التحفظ المحاسبي المحاسبي) أي أن 


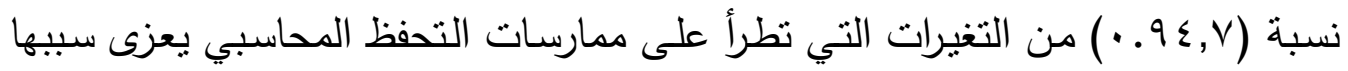
إلى المستحقات غير الاختيارية والمستحقات الاختيارية والمتبقي (r, ه . •) يرجع سببها

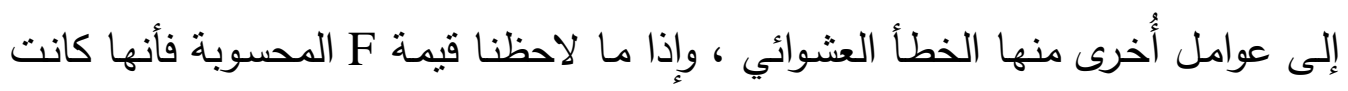

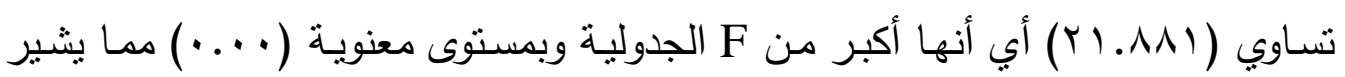
إلى صلاحية النموذج.

تتفق الدراسة مع (Basu,1997) في سرعة استجابة الأرباح للأخبار السيئة (الخسائر المتوقعة) تكون أسرع من استجابتها للأخبار الجيدة (المكاسب المنوقعة) والذي يعد مؤشراً على زيادة في مستوى ممارسات التحفظ المحاسبي حيث تم الاستدلال على لهى ذلك من خلال المقارنة في مستوى ممارسات التحفظ المحاسبي بين الثركات الخاسرة والثركات الرابحة، وتختلف الدراسة مع دراسة (Penman and zhang,2002) في أن إدارة الأرباح يمكنها الاستفادة من ممارسات التحفظ المحاسبي لتكوين احتياطيات

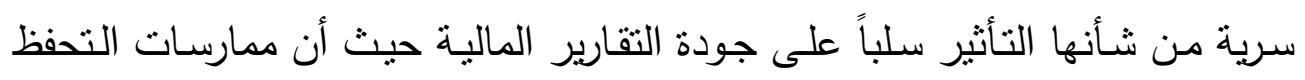
المحاسبي تعمل على النقيض من ممارسات إدارة الأرباح أضافة إلى أن ممارسات التحفظ المحاسبي تعد سياسة وقائية لمقابلة حالة عدم التأكد التي تحبط بيئة الأعمال وهي في نفس الوقت تمثل مبدأ ساند لاستمرارية المنشأة ، وتتقق نتائج الدراسة مع في أن ممارسات التحفظ المحاسبي تعكس سمة هامة في (Watts, 2003a, b) جودة التقارير المالية وتساهم في التخفيف من حدة عدم تماثل المعلومات وتحد من اسلوك الانتهازي للمديرين في الانخراط في ممارسات إدارة الأرباح. وهذا يتفق أيضاً هـ (Lara et al.,2009) ويدفع المديرين للابتعاد عن المشـاريع الخاسرة (ذات صافي القيمة الحالية السالبة) ويساهم في توجيه موارد المنشأة نحو الششاريع الجيدة (ذات صافي القيمة الحالية الموجبة) وينطبق هذا الاتفاق مع دراسة ( Ball and 
وأن الأرباح المتحفظة هي أفضل بكثير من الأرباح غير (Shivakumar,2005 المتحفظـة وتعد سـمة هامـة في جودة التقارير الماليـة وأن التقارير الماليـة لثـركات القطاع العام أعلى جودة من الثقارير المالية لثـركات القطاع الخاص هذه النتيجة منسقة أيضاً مع دراسة (Ferrero,2014) في أن ممارسات التحفظ المحاسبي يؤدي إلى جودة في المستحقات والتي لها الأثر الإيجابي على الأداء المـالي للمننـآت وبالتبعية يؤدي إلى زيادة جودة التقارير المالية، وتتفق الدراسة أيضـاً مع ما توصلت الإهل اليهـ دراسـة (Iatridis,2011) في أن جودة التقارير المالية ترتبط بمستوى تطبيق الممارسات المحاسبية المتحفظة التي من شأنها خفض حدة عدم تماثل المعلومات الأمـر الذي ينـكس إيجابيـاً على أحكام وقـرارات أكثر رشــاً مـن قبـل مستخدمي

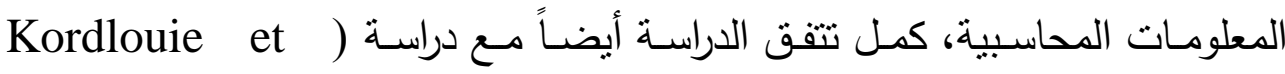
al.,2014) في أن لممارسات ممارسات التحفظ المحاسبي دور هام في جودة التقارير

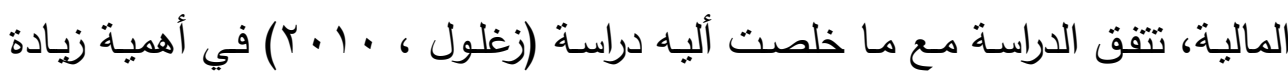
مستوى تطبيق الممارسـات المحاسبية المتحفظة كونها تزيد من موثوقية المعلومات

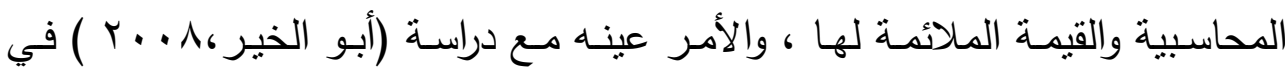
ضرورة التمسك بممارسات ممارسات التحفظ المحاسبي في قياس الأصول وضرورة تمسـك المحاسـبين ومراقبـي الحسـابات والمنظمـين بممارسـات ممارسـات الـتحفظ المحاسبي لأنه لا يرتب عليهم أية مسؤوليات قانونية.

\section{النتائج والتوصيات}

أولاً: النتائج:

1- يوجد تفاوت في مستوى تطبيق الممارسـات المحاسبية في التقارير المالية للشركات المساهمة المقيدة في سوق العراق للأوراق المالية. 
ץ- يوجد تفاوت في مستوى جودة التقارير المالية للثركات المساهمة المقيدة في سوق العراق للأوراق المالية. r-توصل الباحث من خلال اختبار العلاقة ما بين ممارسات التحفظ المحاسبي لوني

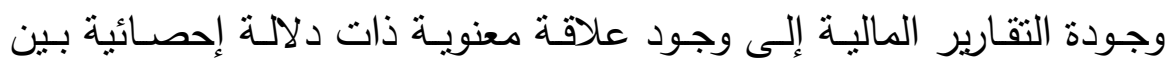
ممارسات التحفظ المحاسبي وجودة التقارير المالية وذلك من خلال استخدام المدخل الملائم الذي يمكنه نوضيح العلاقة بينهما. ثانياً: التوصبات الندل التاث

1- يوصي الباحث مجلس المعايير المحاسبة والرقابية وهيئة سوق العراق للأوراق

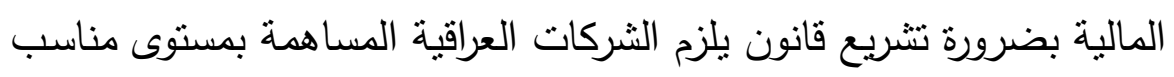

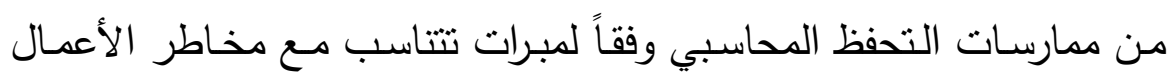

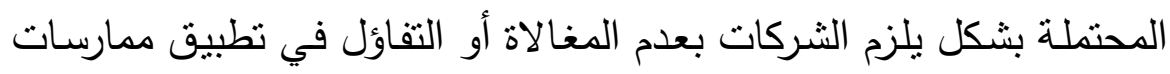

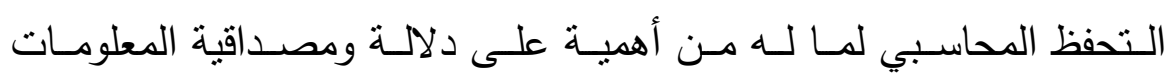

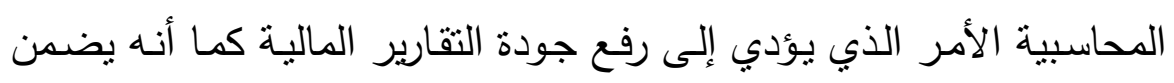

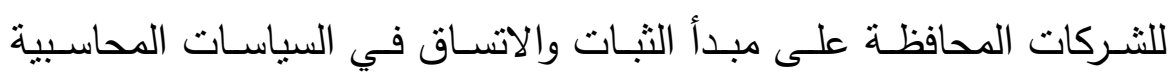
وعرض المعلومات المحاسبية عند إعداد الثقارير المالية.

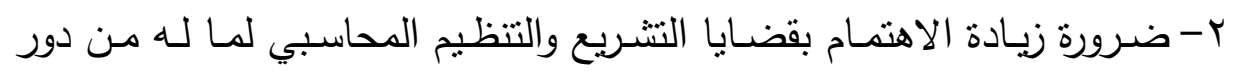
كبير في ضبط تطبيق الممارسات المحاسبية المتحفظة بشكل سليم وتزيد من جودة الثقارير المالية كتثريع المزيد من القوانين المتعلقة بحوكمة الثركات وتفعيل دور لجان التدقيق وحماية المستثر .

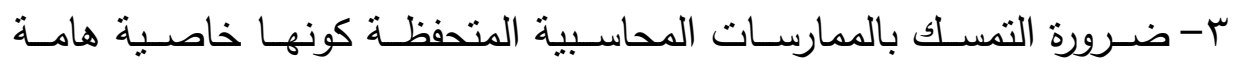

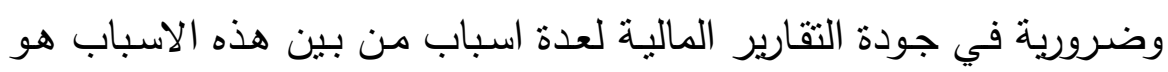

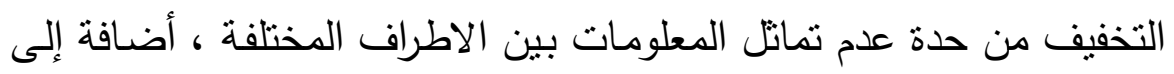

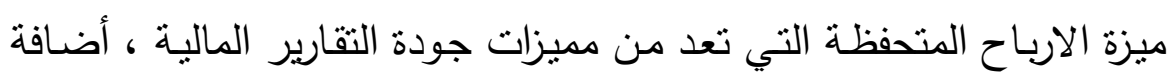

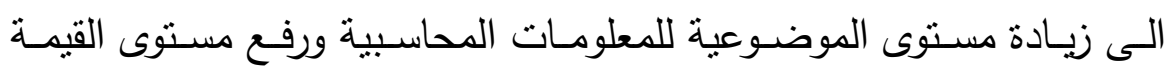


الملائمة للمعلومات المحاسبية كل ذلك من شانه أن يعزز منفعة المعلومات

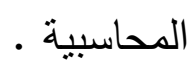

\section{قائمة المراجع}

\section{اولا : مراجيع بالغة العربية :}

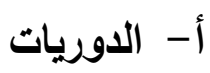

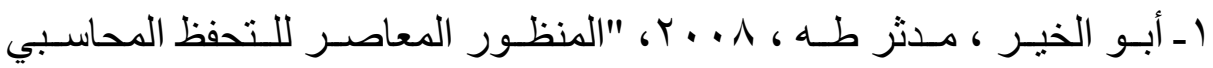

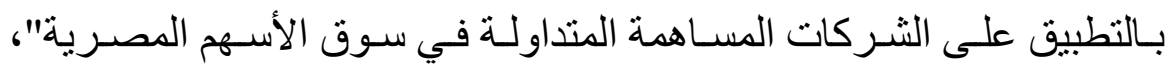

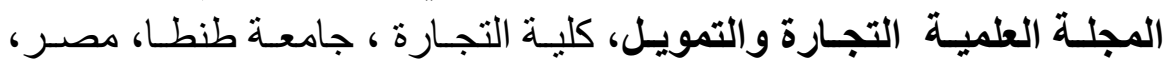

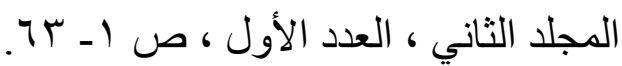

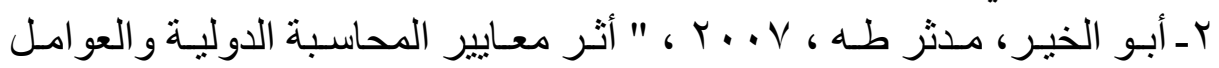

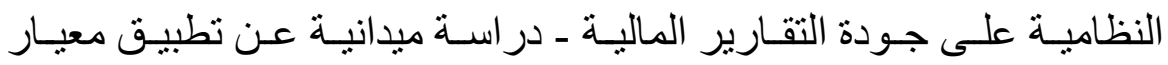

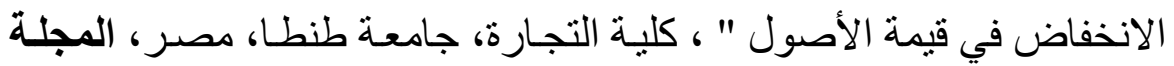

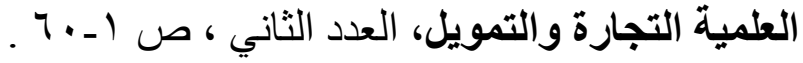

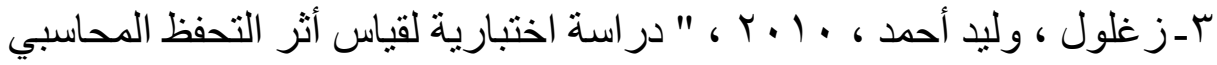

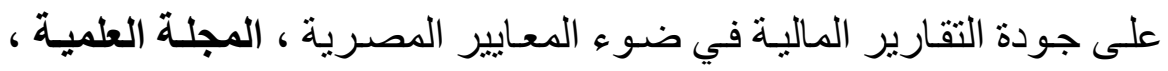

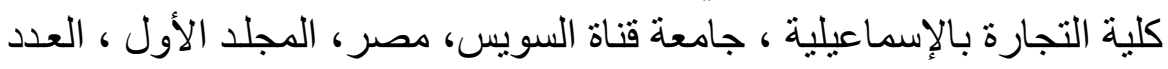

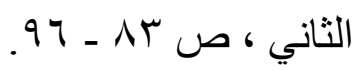

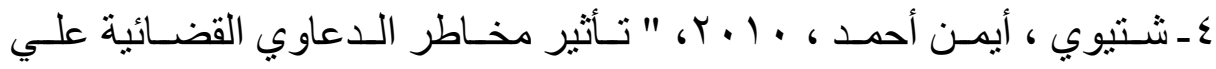

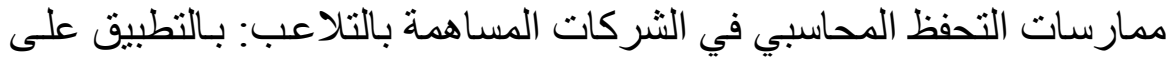

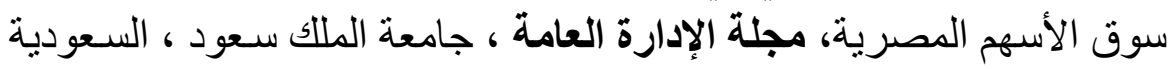

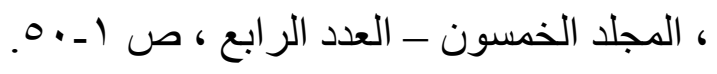

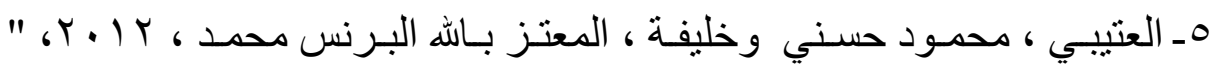

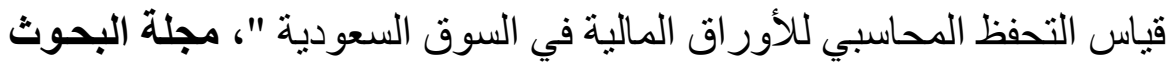

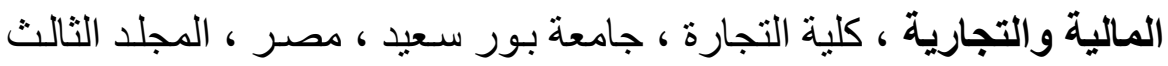

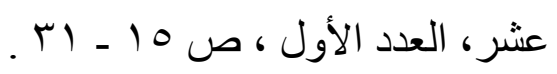

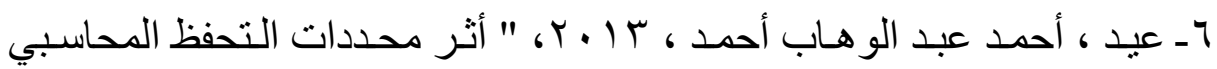

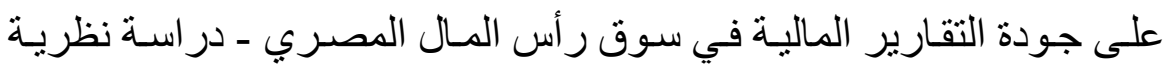

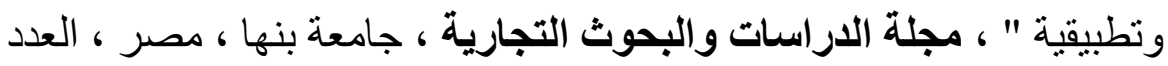

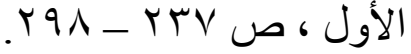

$$
\begin{aligned}
& \text { بـ الرسائل العلمية }
\end{aligned}
$$




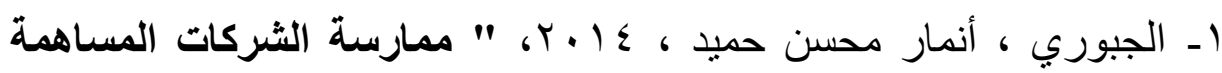
للتحفظ المحاسبي وأنعكاسها على القرارات الاقتصادية لمستخدمي القوائم

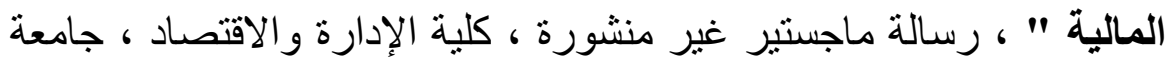

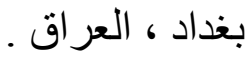

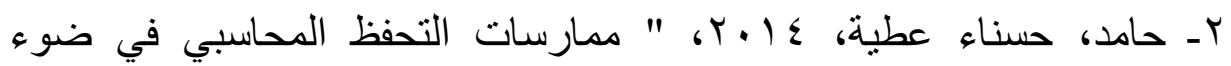
الاعتبار ات الضريبية و أثره على دلالة القوائم المالية ـ در اسة تطبية الطبيقية في بيئة الأعمال المصرية "، رسالة دكتوراه غير منشورة، كلية التجارة، جامعة دالهاه المنصورة، مصر.

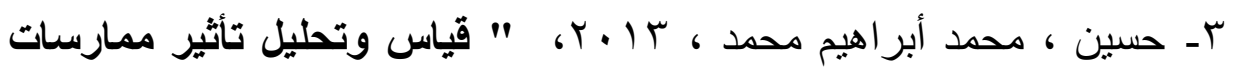

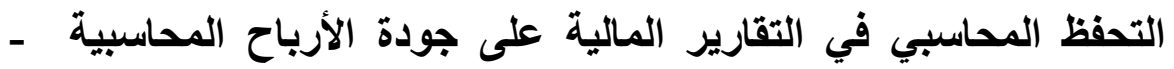

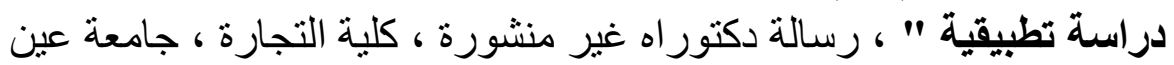
شمس ، مصر.

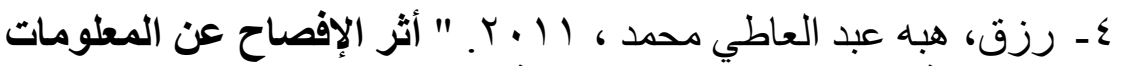

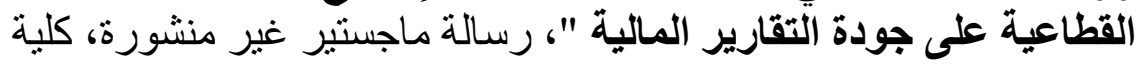

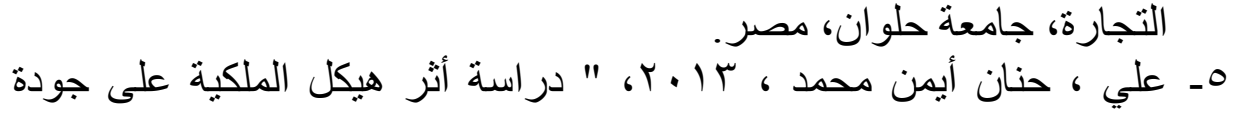

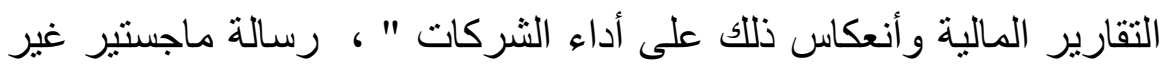

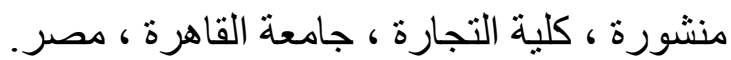
ثنانيا : مراجع باللغة الانجليزية :

1- Ball, R. \& Shivakumar, L., 2005, "The role of accruals in asymmetrically timely gain and loss recognition", Graduate School of Business, University of Chicago, U.S.A, Journal of Accounting Research, Vol.39, pp.207-242..

2- Ball, R., Kothari, S. P., \& NIKOLAEV, Valeri .V,2007, "Econometrics of the Basu asymmetric timeliness coefficient and accounting conservatism", University of Chicago Booth School of Business ,U.S.A, Working Paper, No. 09-16,pp.1-31, Available at: http://ssrn.com. 
3- Basu, S., 1997, "The conservatism principle and the asymmetric timeliness of earnings", Journal of Accounting and Economics, Baruch College, CUNY, U.S.A, Vol. 24, pp.3-37.

4- Beaver, W. H. \& Ryan, S. G., 2000," Biases and lags in book value and their effects on the ability of the book-to-market ratio to predict book return on equity", School of Business, University of Chicago Stable ,U.S.A, Journal of Accounting Research , Vol.38,No.1, pp. 127-148.

5-Dechow, P. M., Sloan, R. G., \& Sweeney, A. P. ,1995, "Detecting earnings management, University of Pennsylvania ,U.S.A ,Accounting review, Vol. 70, No. 2 , PP: 193-225.

6- Ferrero, J. M., 2014,"Consequences of financial reporting quality on corporate performance. Evidence at the international level", University of Salamanca, Spain, Estudios de Economía, Vol.41, No.1, pp.49-88.

7- Givoly, D. \& Hayn, C., 2000, "The changing time-series properties of earnings, cash flows and accruals: Has financial reporting become more conservative?", School of Management, University of California,U.S.A, Journal of Accounting and Economics , Vol.29,No.3, pp.287-320.

8- Iatridis, G. E., 2011, "Accounting disclosures, accounting quality and conditional and unconditional conservatism", University of Thessaly, Greece International Review of Financial Analysis, Vol. 20, No.2, pp.88-102.

9- Jonas, G. J. \& Blanchet, J., 2000, "Assessing quality of financial reporting".U.S.A, Accounting Horizons, vol.14 No. 3, pp. 353-363.

10-Lafond, R. \& Roychowdhury, S., 2008, "Managerial ownership and accounting conservatism", University of Chicago, U.S.A, Journal of Accounting Research, Vol.46, NO.1, pp.101-135.

11-Lara, J. M. G. A., Osma, B. G. A. \& Penalva, F., 2009, "Accounting conservatism and corporate governance", Review of Accounting Studies, University of Carlos III de Madrid, Spain Vol.14, No.1, pp.161-201. 
12- Penman, S. H. \& Zhang, X.-J., 2002, "Accounting conservatism, the quality of earnings, and stock returns". The Accounting Review, School of Business, Columbia University, U.S.A, Vol. 77, No. 2, pp. 237-264.

13- Watts, R. L., 2003a, "Conservatism in accounting part I: Explanations and implications", School of Business Administration, University of Rochester,U.S.A, Accounting horizons, Vol.17, No.3, pp. 207-221.

14- Watts, R. L., 2003b, "Conservatism in accounting part II: Evidence and research opportunities", School of Business Administration, University of Rochester,U.S.A, Accounting horizons, Vol.17, No.4, pp.287-301.

15- Wang, R. Z., 2009,"Accounting Conservatism", Doctor of Philosophy in accounting thesis, School of accounting and commercial law, Victoria University of Wellington, New Zealand.

1- IFRS Foundation, 2010, U.K, Available at: http://www.iasplus.com.

2- Iraqi Securities Commission Available at: http://www.isc.gov.iq. 